\title{
Clearance of germ granules in the soma Asako Sugimoto
}

\author{
Address: Laboratory for Developmental Genomics, RIKEN Center for Developmental Biology, 2-2-3 Minatojima-Minamimachi, Chuo-ku, \\ Kobe 650-0047, Japan \\ Email: sugimoto@cdb.riken.jp
}

FI000 Biology Reports 2009, I:49 (doi:I0.34I0/BI-49)

The electronic version of this article is the complete one and can be found at: http://FI000.com/Reports/Biology/content/I/49

\begin{abstract}
Germ granules are ribonucleoprotein complexes specifically segregated into germ cell lineages in diverse organisms. Recent studies indicate that multiple mechanisms are involved in the clearance of germ granules and their components in somatic cells in Caenorhabditis elegans embryos.
\end{abstract}

\section{Introduction and context}

In many organisms, cells of the germ lineage contain large ribonucleoprotein (RNP) complexes, generally called germ granules or germ plasm, which are believed to function in post-transcriptional gene regulation to contribute to germ cell specification and/or differentiation [1]. Considering the importance of the binary decision between germ versus soma, specific segregation of germ granules into germ lineage cells must be strictly regulated.

The nematode Caenorhabditis elegans provides an excellent model system to study germ granule segregation in early development: in C. elegans embryos, the germ lineage is separated from the soma through a series of four asymmetric cell divisions, during which germ granules (P granules) are sequentially partitioned into the germ lineage cells (Figure 1). In recent years, components of $\mathrm{P}$ granules have been identified, and currently over 20 proteins (mostly RNA binding proteins) and some specific maternal mRNAs are known [2]. Among the protein components, P granule abnormality (PGL) proteins (PGL-1 [3] and PGL-3 [4]) and germ-line helicase (GLH) proteins (Drosophila vasa homologs) [5-7] are present on P granules throughout development, whereas other components are transiently detected on $P$ granules at certain periods of embryogenesis.

Live imaging of early embryogenesis revealed that segregation of $\mathrm{P}$ granules into germ lineage cells is achieved by a combination of mechanisms [8]: firstly,
$\mathrm{P}$ granules are swept by the cytoplasmic streaming towards the side of the future germ blastomeres; secondly, P granules rapidly disappear in the cytoplasm destined for the somatic daughter and the resultant somatic cells. These observations led to a model in which there is a $\mathrm{P}$ granule stabilizing/destabilizing gradient along the axis of each germ lineage division, and the remnant $P$ granules in the soma are rapidly disassembled and/or degraded [8].

\section{Major recent advances}

Recent studies have uncovered some of the molecular details of the clearance mechanisms of $\mathrm{P}$ granule components from the soma in early C. elegans embryos.

DeRenzo et al. [9] showed that transient $\mathrm{P}$ granule components that have CCCH fingers (PIE-1, POS-1, and MEX-1) are degraded by the ubiquitin-proteasome system [10]. They observed that ZIF-1 (zinc finger interacting factor 1 ) directly binds to the $\mathrm{CCCH}$ finger of these proteins and targets them for soma-specific degradation (Figure 1) via a cullin-dependent pathway that includes the ECS family of E3 ubiquitin ligases (elongin C, CUL-2, RBX-1) and the E2 ubiquitinconjugating enzyme UBC5. The soma-specific activation of the ZIF-1-dependent degradation system requires MEX-5 and MEX-6, the redundant CCCH finger proteins that accumulate in somatic cells; whereas the low levels of MEX-5/6 in germline blastomeres are achieved by the function of PAR-1 serine/threonine protein kinase [10]. 
Figure I. Phenotypes of the mutants defective in $\mathbf{P}$ granule degradation in the soma

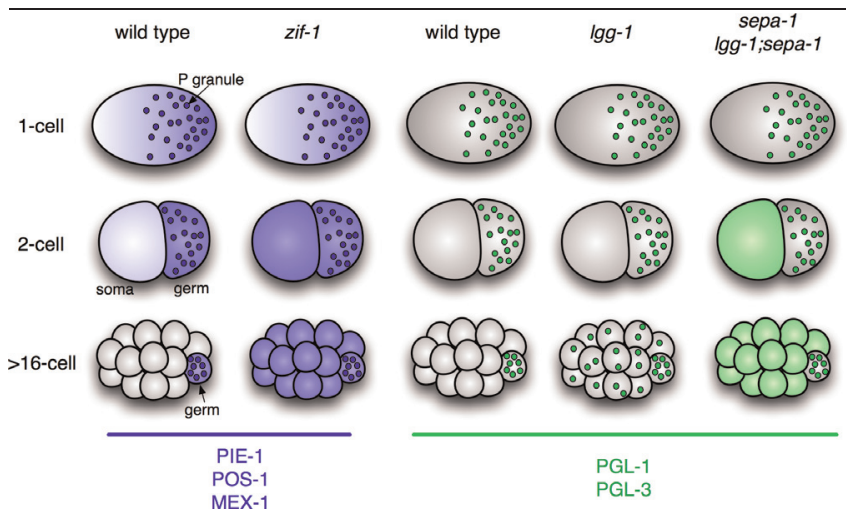

Blue: The $\mathrm{CCCH}$ finger proteins (PIE-I, POS-I, MEX-I). These proteins transiently localize on $\mathrm{P}$ granules and are also dispersed in the germ cell cytoplasm in the wild type. In zif-I (RNAi) embryos, their degradation in the soma is defective (asymmetry of PIE-I in the one-cell embryo is unaffected). Green: PGL-I and PGL-3, constitutive components of P granules. In the wild type, their localization is limited to $P$ granules in the germ lineage. In autophagy mutants (for example, Igg-I), ectopic PGL granules are formed in somatic cells after about the I6-cell stage. In the sepa-I mutants, PGL-I and PGL-3 are dispersed in the somatic cytoplasm.

Interestingly, the loss of ZIF-1 function does not affect the asymmetric accumulation of PIE-1 in the cytoplasm on the side of future germline blastomeres in one-cell stage embryos (Figure 1), suggesting that another mechanism exists for PIE-1 exclusion from the somatic side, other than cullin-dependent degradation. Using quantitative live-cell imaging of GFP::PIE-1 and mathematical modeling, Daniels et al. [11] recently proposed a novel model in which the lower diffusion coefficient of PIE-1 on the posterior side (future germ blastomeres) than on the anterior side leads to a stable protein gradient across the zygotes. Based on the correlation of the posterior distribution of P granules and PIE-1, they went on to speculate that $P$ granules may act, at least in part, to slow the diffusion of PIE-1 through the surface association, possibly via their RNA components. Further studies are awaited to experimentally examine this hypothesis. It will also be of interest to see whether this heterogeneous reaction/diffusion model contributes to the PIE-1 exclusion from the somatic cells after the two-cell stage, in combination with the proteasomedependent degradation.

Since the loss of ZIF-1 function does not affect the somatic degradation of other P granule components that do not contain CCCH fingers (for example, PGL-1), the ZIF-1-dependent degradation system accounts for only part of the P granule clearance in the soma. The question remains, however, as to how other P granule components degraded.

Recent work by Zhang et al. [12] provided evidence that autophagy is involved in the elimination of some of the constitutive P granule components (PGL-1 and PGL-3) in somatic cells during C. elegans embryogenesis (Figure 1). Autophagy is the intracellular catabolic mechanism for degradation of cellular components via the formation of a double-membrane structure called the autophagosome, which delivers a portion of the cytoplasm or organelles to lysosomes for degradation [13]. Zhang et al. [12] found that GFP::PGL-1 ectopically formed aggregates (PGL granules) in the somatic cells in embryos with mutations of the autophagy pathway (lgg-1 (homolog of yeast $\operatorname{atg} 8), \operatorname{lgg}-2 / \operatorname{atg} 8, \operatorname{lgg}-3 / \operatorname{atg} 12, \operatorname{atg}-3, \operatorname{atg}-4.1$, and atg-7). They further genetically identified SEPA-1 (suppressor of ectopic P granule in autophagy) mutants; SEPA-1 is required for the formation of PGL granules in these autophagy mutants. In sepa-1 mutant embryos, both PGL-1 and PGL-3 are diffused and persistent in the somatic cytoplasm (Figure 1); thus, SEPA-1 is required for PGL protein degradation in the wild-type environment. SEPA-1 directly binds to PGL-3 and self-aggregates; PGL-1 appears to be recruited to these aggregates through binding with PGL-3. Since SEPA-1 also binds to LGG-1, SEPA-1 appears to function as an adaptor protein that delivers PGL proteins to the autophagy pathway for their degradation.

Although PGL granules are formed in somatic cells in autophagy mutant embryos after about the 16-cell stage, these somatic cells show no sign of transformation into germ cell fates, suggesting that these ectopic granules cannot function as germ cell fate determinants. Indeed, other constitutive P granule components (GLH-1 and GLH-4) are not detectable on these granules. Whether GLH proteins and other P granule components undergo controlled degradation in the soma is currently unclear.

\section{Future directions}

Collectively, these studies revealed the multiple layers of mechanisms for the clearance of $\mathrm{P}$ granules and their components in somatic cells during early C. elegans embryogenesis. Since remnants of functional germ granules in the soma would be potentially detrimental to animal development, mechanisms for the rapid and thorough removal of germ granules might be necessary. Obviously, there are still many unanswered questions. How are other P granule components (for example, GLH proteins) cleared in somatic cells and are other specific E3 ubiquitin ligases involved? What are the molecular mechanisms of $\mathrm{P}$ granule stabilization in the germ lineage and destabilization in the soma? It will also be 
interesting to see whether the mechanisms of germ granule clearance from the soma described here are utilized by other organisms.

\section{Abbreviations}

atg-3, autophagy 3; CUL-2, cullin-2; GFP, green fluorescent protein; GLH, germ-line helicase; LGG-1, LC3, GABARAP and GATE-16 family member 1; MEX-1, muscle excess 1; PGL-1, P granule abnormality 1; PAR-1, abnormal embryonic partitioning of cytoplasm 1; PIE-1, pharynx and intestine in excess protein 1; POS-1, posterior segregation 1 ; RBX-1, ring box 1; RNP, ribonucleoprotein; SEPA-1, suppressor of ectopic P granule in autophagy 1 ; ZIF-1, zinc finger interacting factor 1 .

\section{Competing interests}

The author declares that she has no competing interests.

\section{References}

I. Seydoux G, Braun RE: Pathway to totipotency: lessons from germ cells. Cell 2006, 127:891-904.

2. Strome S: Specification of the germ line. In WormBook. Edited by The C. elegans Research Community. Jul 28 2005. [http://www. wormbook.org/chapters/www_specgermline/specgermline.html].

3. Kawasaki I, Shim YH, Kirchner J, Kaminker J, Wood WB, Strome S: PGL-I, a predicted RNA-binding component of germ granules, is essential for fertility in C. elegans. Cell 1998, 94:635-45.

4. Kawasaki I, Amiri A, Fan Y, Meyer N, Dunkelbarger S, Motohashi T, Karashima T, Bossinger O, Strome S: The PGL family proteins associate with germ granules and function redundantly in Caenorhabditis elegans germline development. Genetics 2004, 167:645-61.

5. Kuznicki KA, Smith PA, Leung-Chiu WM, Estevez AO, Scott HC, Bennett KL: Combinatorial RNA interference indicates GLH-4 can compensate for GLH-I; these two P granule components are critical for fertility in C. elegans. Development 2000, 1 27:2907-16.

6. Orsborn AM, Li W, McEwen TJ, Mizuno T, Kuzmin E, Matsumoto K, Bennett KL: GLH-I, the C. elegans $\mathbf{P}$ granule protein, is controlled by the JNK KGB-I and by the COP9 subunit CSN-5. Development 2007, 134:3383-92.

7. Spike C, Meyer N, Racen E, Orsborn A, Kirchner J, Kuznicki K, Yee C, Bennett K, Strome S: Genetic analysis of the Caenorhabditis elegans GLH family of P-granule proteins. Genetics 2008, I78:1973-87.

8. Hird SN, Paulsen JE, Strome S: Segregation of germ granules in living Caenorhabditis elegans embryos: cell-type-specific mechanisms for cytoplasmic localisation. Development 1996, 122:1303-12

9. DeRenzo C, Reese KJ, Seydoux G: Exclusion of germ plasm proteins from somatic lineages by cullin-dependent degradation. Nature 2003, 424:685-9.

FI000 Factor 4.9 Must Read

Evaluated by Jonathan Hodgkin II Aug 2003, Jane Hubbard I4 Aug 2003, Zhen-Qiang Pan 16 Oct 2003

10. Ravid $\mathrm{T}$, Hochstrasser $\mathrm{M}$ : Diversity of degradation signals in the ubiquitin-proteasome system. Nat Rev Mol Cell Biol 2008, 9:679-90.

II. Daniels BR, Perkins EM, Dobrowsky TM, Sun SX, Wirtz D: Asymmetric enrichment of PIE-I in the Caenorhabditis elegans zygote mediated by binary counterdiffusion. J Cell Biol 2009, I84:473-9.

12. Zhang Y, Yan L, Zhou Z, Yang P, Tian E, Zhang K, Zhao Y, Li Z, Song B, Han J, Miao L, Zhang H: SEPA-I mediates the specific recognition and degradation of $P$ granule components by autophagy in C. elegans. Cell 2009, 136:308-21.

FI000 Factor 4.8 Must Read

Evaluated by Eric Baehrecke 26 Feb 2009, Phillip Newmark 26 Feb 2009

13. Mizushima N: Autophagy: process and function. Genes Dev 2007, 2I:286I-73. 